\title{
Genetic Variation and Population Structure in Jamunapari Goats Using Microsatellites, Mitochondrial DNA, and Milk Protein Genes
}

\author{
P. K. Rout, ${ }^{1}$ K. Thangraj, ${ }^{2}$ A. Mandal, ${ }^{1}$ and R. Roy $^{1}$ \\ ${ }^{1}$ Central Institute for Research on Goats, Makhdoom, Farah, Mathura 281122, India \\ ${ }^{2}$ Centre for Cellular and Molecular Biology, Uppal Road, Hyderabad 500007, India
}

Correspondence should be addressed to P. K. Rout, rout_ctc@hotmail.com

Received 2 March 2011; Accepted 25 October 2011

Academic Editor: Martien Groenen

Copyright ( 2012 P. K. Rout et al. This is an open access article distributed under the Creative Commons Attribution License, which permits unrestricted use, distribution, and reproduction in any medium, provided the original work is properly cited.

\begin{abstract}
Jamunapari, a dairy goat breed of India, has been gradually declining in numbers in its home tract over the years. We have analysed genetic variation and population history in Jamunapari goats based on 17 microsatellite loci, 2 milk protein loci, mitochondrial hypervariable region I (HVRI) sequencing, and three Y-chromosomal gene sequencing. We used the mitochondrial DNA (mtDNA) mismatch distribution, microsatellite data, and bottleneck tests to infer the population history and demography. The mean number of alleles per locus was 9.0 indicating that the allelic variation was high in all the loci and the mean heterozygosity was 0.769 at nuclear loci. Although the population size is smaller than 8,000 individuals, the amount of variability both in terms of allelic richness and gene diversity was high in all the microsatellite loci except ILST 005. The gene diversity and effective number of alleles at milk protein loci were higher than the 10 other Indian goat breeds that they were compared to. Mismatch analysis was carried out and the analysis revealed that the population curve was unimodal indicating the expansion of population. The genetic diversity of Y-chromosome genes was low in the present study. The observed mean $M$ ratio in the population was above the critical significance value $(\mathrm{Mc})$ and close to one indicating that it has maintained a slowly changing population size. The mode-shift test did not detect any distortion of allele frequency and the heterozygosity excess method showed that there was no significant departure from mutation-drift equilibrium detected in the population. However, the effects of genetic bottlenecks were observed in some loci due to decreased heterozygosity and lower level of $\mathrm{M}$ ratio. There were two observed genetic subdivisions in the population supporting the observations of farmers in different areas. This base line information on genetic diversity, bottleneck analysis, and mismatch analysis was obtained to assist the conservation decision and management of the breed.
\end{abstract}

\section{Introduction}

Genetic diversity, the primary component of adaptive evolution, is essential for the long-term survival probability of a population [1-4]. Genetic diversity within domesticated species depends on several factors such as changing agricultural practices, breed replacement, and cross breeding. Genetic diversity has been analysed by using protein polymorphism, mitochondrial diversity, and microsatellite marker in both domestic and wild species [4-11]. Jamunapari goat, the majestic milk-producing goat breed of India, has suffered a reduction in numbers in its home tract $[12,13]$ and is considered as an endangered breed [14]. The Indian Jamunapari goat is one of the ancestors of the American Nubian and has been used in India and adjacent countries as an improver breed. The breed possesses several unique characteristics such as higher kidding rate despite its large body size. The breed inhabits isolated ravines in the Chakarnagar area of Etawah (Uttar Pradesh, India) (Figure 1), and geographical isolation has contributed towards the evolution of this unique breed. The breed is gradually declining in number due to land reclamation, decrease in grazing area, breed replacement, and the population size is less than 8,000 [12]; therefore, there is an urgent need to define strategies for conservation of this breed in its natural habitat. 


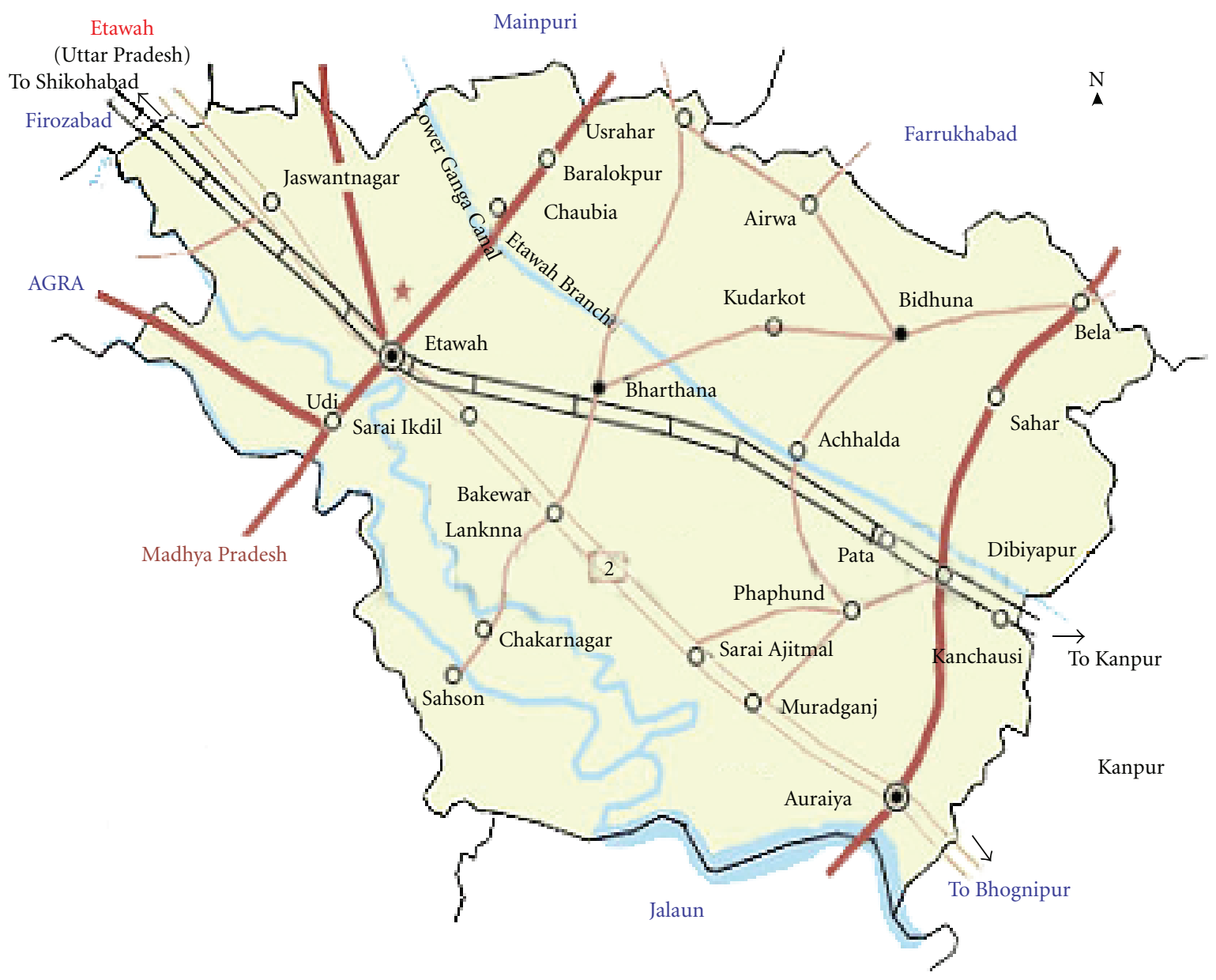

FIGURE 1: Maps of Chakarnagar (Etawah, UP) showing the home tract of Jamunapari goats.

In this study, we sample the Jamunapari goat population to analyse the genetic variation due to locus-specific events (selective sweep) as well as genome wide events (bottlenecks). Microsatellite markers are highly polymorphic and have been extensively used for breed diversity analysis. Mitochondrial DNA (mt DNA) and Y-chromosome region are usually sensitive to genetic drift and can be useful for detecting effects of bottlenecks in the population. Nonneutral markers are also being used to analyse population diversity, and milk protein gene has been used as the region is directly involved for the survival of the individual and under strong selective pressure. By integrating data from multiple markers, we provide the possible factors affecting the genetic consequence of population reduction in this breed.

\section{Materials and Methods}

Fifty blood samples were collected in 10 villages in which the breed has a major concentration. Samples were collected from the individuals exhibiting typical breed characteristics such as white colour, Roman nose, and pendulous ear (farmers are not selecting for these traits) and at least two samples were collected from each village. An effort was made to collect samples from unrelated individuals based on informa- tion provided by farmers. The breeding buck is available with one or two farmers in every village, and some farmers also maintain breeding bucks during breeding season, disposing of them after the breeding season. Blood samples were collected from each animal using EDTA vacutainer and stored at $-20^{\circ} \mathrm{C}$ till further use.

Microsatellite analysis was carried out to test for signatures of recent population bottlenecks in Jamunapari goats. This analysis was carried out on 49 DNA samples with 17 microsatellite markers (Table 1) as reported by Rout et al. [11]. For these 17 loci, genetic variation was quantified using measures of the total number of alleles, number of polymorphic loci, observed and expected heterozygosity per locus, and allelic richness using GENEPOP (Version 3.4; [15]),

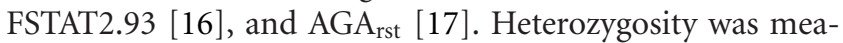
sured as the mean observed heterozygosity (Ho) and the mean expected heterozygosity $\left(\mathrm{H}_{\mathrm{E}}\right)$ based on Hardy-Weinberg assumptions. We tested genotypic linkage disequilibrium between all pairs of loci in each population with GENEPOP (Version 3.4; [15]) based on Markov chain method with 10,000 iterations and 100 batches. We also used FSTAT software to assess 95\% confidence intervals of Weir and Cockerham's $\mathrm{f}$, which measures deviation from the Hardy-Weinberg equilibrium (HWE) for populations and 
TABLE 1: Microsatellite markers and chromosomal location, total number of alleles and genetic diversity in the Jamunapari goats.

\begin{tabular}{|c|c|c|c|c|c|c|}
\hline Markers & Chromosome number & Observed number of alleles & Allele size range (bp) & Gene diversity & Allelic richness & $\mathrm{F}_{\mathrm{IS}}$ \\
\hline BM4621 & 6 & 15 & $106-140$ & 0.862 & 15.00 & 0.652 \\
\hline NRAMP & 2 & 10 & $224-248$ & 0.807 & 10.00 & 0.554 \\
\hline OarAE101 & 6 & 8 & $92-108$ & 0.809 & 8.00 & 0.555 \\
\hline IDVGA7 & 25 & 15 & $210-240$ & 0.890 & 15.00 & 0.573 \\
\hline ILSTS005 & 10 & 3 & $178-188$ & 0.497 & 3.00 & 0.235 \\
\hline BM6526 & 27 & 9 & $154-178$ & 0.801 & 9.00 & 0.500 \\
\hline ETH225 & 14 & 6 & $140-152$ & 0.703 & 6.00 & 0.089 \\
\hline OarHH56 & 23 & 9 & $152-168$ & 0.818 & 9.00 & 0.560 \\
\hline INRABERN192 & 7 & 10 & 178-208 & 0.823 & 10.00 & 0.417 \\
\hline OarFCB48 & 17 & 8 & $150-164$ & 0.831 & 8.00 & 0.351 \\
\hline OarHH62 & 20 & 5 & $108-118$ & 0.719 & 5.00 & 0.499 \\
\hline TGLA40 & & 10 & $174-198$ & 0.782 & 10.00 & 0.540 \\
\hline BM143 & 6 & 8 & $96-118$ & 0.741 & 8.00 & 0.514 \\
\hline SRCRSP 5 & 21 & 8 & $160-178$ & 0.794 & 8.00 & 0.748 \\
\hline SRCRSP6 & 19 & 10 & $138-158$ & 0.680 & 10.00 & 0.530 \\
\hline SRCRSP9 & & 11 & $120-144$ & 0.877 & 11.00 & 0.247 \\
\hline SRCRSP10 & 8 & 9 & $260-276$ & 0.836 & 9.00 & 0.785 \\
\hline
\end{tabular}

corresponds to Wright's within-population inbreeding coefficient $\mathrm{F}_{\mathrm{IS}}$.

Milk protein genes, which are expected to be nonneutral markers, were also used to analyse the population variability. Two milk protein genes, namely, $\beta-L G$ gene and CSN1S1 $\left(\alpha \mathrm{s}_{1}\right.$-casein) were analysed using PCR-RFLP to observe genetic variability in 35 individuals. The $\alpha \mathrm{s}_{1}$-casein (CSN1S1) gene produced an amplified fragment of $223 \mathrm{bp}$ which was digested with the XmnI restriction enzyme. The $\beta$-LG gene produced an amplified product of $426 \mathrm{bp}$, and RFLP analysis was carried out with the SacII restriction enzyme. The PCR-RFLP analysis was carried out as described by Kumar et al. $[18,19]$, and the data were analysed separately for mean number of alleles, expected heterozygosity and Hardy-Weinberg equilibrium (HWE) using POPGENE software [20].

mtDNA HVRI sequencing was carried out as described by Joshi et al. [10]. Four hundred and fifty-seven base pairs from the mtDNA HVRI regions of 50 individuals were aligned using CLUSTAL X. We used mismatch distribution [21] to analyse the population expansion as implemented in ARLEQUIN 3.1 [22]. Fu's F value was calculated from mtDNA haplotypes to test for deviations from neutral equilibrium condition [23]. The qualitative and quantitative aspect of the population's genetic history may be uncovered by the analysis of frequency distributions of pairwise sequence mismatches. Mismatch analysis (the distribution of all pair-wise nucleotide differences between sequences) was carried out to test the deviation of the observed data from neutral predictions expected in constant-sized populations.

Genetic divergence was analysed by selecting three primers from ovine male-specific region (AMLEY, SRY, and ZFY gene) [24]. PCR was carried out in a $50 \mu \mathrm{L}$ reaction volume containing $100 \mathrm{ng}$ of DNA, $20 \mathrm{pM}$ of each primer, $200 \mu \mathrm{M}$ of dNTP, $2 \mathrm{mM} \mathrm{Mgcl}_{2}$, and \%U of Taq DNA polymerase (New India Biolab, MA, USA). The samples were subjected to seq- uencing after purifying the PCR product by gene elute PCR clean up kit. Individual PCR amplified products were subjected to sequencing in 12 samples. PCR products were sequenced on both the strands directly using $50 \mathrm{ng}(2.0 \mu \mathrm{L})$ of PCR product and $4 \mathrm{pM}(1.0 \mu \mathrm{L})$ of primer, $4 \mu \mathrm{L}$ of Big Dye Terminator ready reaction kit (Perkin Elmer, Foster City, USA), and $3.0 \mu \mathrm{L}$ of double distilled water to adjust the volume to $10.0 \mu \mathrm{L}$. Cycle sequencing was carried out in a Gene Amp 9600 thermal cycler (Perkin Elmer) employing the PCR conditions. Extended products were purified by alcohol precipitation followed by washing with $70 \%$ alcohol. Purified samples were dissolved in $10 \mu \mathrm{L}$ of $50 \% \mathrm{Hi}$-Di formamide and analysed in an ABI 3700 automated DNA Analyzer (Perkin Elmer, USA). Nucleotide diversity, expected heterozygosity, Tajima's D, and Fu's Fs values were estimated in ARLEQUIN 3.1 [22].

Genetic bottleneck was detected using microsatellite data by three approaches, heterozygote excess, mode-shift, and $\mathrm{M}$ ratio test. We first used the $M$ ratio (the mean ratio of the number of alleles to total range in allele size) [25] as implemented in $\mathrm{AGA}_{\mathrm{rst}}$ [17], because of its consistent performance in identifying populations with known bottlenecks. $M$ ratio calculates the changes that occur after a bottleneck in the distribution of allele sizes relative to the number of alleles in a population. It has been established that an $\mathrm{M}$ ratio less than 0.71 signifies a bottleneck [25].

The BOTTLENECK programme [26] was used as an alternative measure of genetic bottlenecks to test for excess gene diversity relative to that expected under mutation-drift equilibrium. The heterozygosity excess method exploits the fact that allele diversity is reduced faster than heterozygosity during a bottleneck, because rare alleles are lost rapidly and have little effect on heterozygosity, thus producing a transient excess in heterozygosity relative to that expected in a population of constant size with the same number of alleles 
$[26,27]$. To determine the population "genetic reduction signatures" characteristic of recent reductions in effective population size (Ne), the Wilcoxon's heterozygosity excess test [26] and the allele frequency distribution mode shift analysis [28] were performed using BOTTLENECK [26]. The heterozygosity excess method was used to analyse the population, and the data for the heterozygosity excess test were examined under the two-phased model (TPM; 95\% stepwise mutation model with 5\% multistep mutations and a variance among multiple steps of 12), which is considered best for microsatellite data $[26,29]$. We also analysed the allele frequency distribution for gaps. A qualitative descriptor of allele frequency distribution (the mode-shift indicator), which is reported to discriminate between bottlenecked and stable population [28], was obtained using the programme BOTTLENECK.

We used an individual-based clustering approach (STRUCTURE 2.1, [30]) to determine the most likely number of genetic clusters $(k)$ in the Jamunapari populations. STRUCTURE software sorts individual genotypes into clusters that maximize the fit of the data to theoretical expectation. Based on preliminary analyses, we evaluated the likelihood of $k=2$ and $k=3$, with 5 runs performed for each $k$, and a burn-in length of 500,000 and 100,000 MCMC replicates for each run. We assumed an admixture model and correlated allele frequencies among populations [30].

\section{Results and Discussion}

The markers with their chromosome number, number of alleles identified, and allele size range have been described in Table 1. Among the polymorphic markers, BM4621 and IDVGA7 showed highest number of alleles (15) at each locus. The number of alleles per microsatellite marker was above 6 for all markers except for ILSTS005 and Oar HH62. The total number of alleles was 153 over the 17 loci. The allelic richness ranged from 3.00 to 15.00 across the microsatellite markers (Table 1) and the mean number of alleles per locus was 9.0. Allelic richness was identical to allele frequency implying that there was no bias based on sample size. The average gene diversity ranged from 0.489 to 0.866 over the loci. The mean expected and observed heterozygosity was 0.769 and 0.386 (Table 1). All the loci showed higher gene diversity than ILSTS005 in the analysed samples. The high mean number of alleles per locus and expected heterozygosities indicated that the overall gene diversity was high in the population. Heterozygosity and allele number are aligning with high diversity score in the population. Takezaki and Nei (1996) suggested that microsatellite loci can be included diversity analysis having heterozygosity from 0.3 to 0.8 in the population. Two loci departed significantly from the Hardy-Weinberg equilibrium (HWE). In the analysed samples, 18 microsatellite locus pairs demonstrated linkage disequilibrium (LD) with $P$ value $<0.05$. The LD was significant in $13.21 \%$ of the locus pair combinations in the population. The overall excess of homozygosity for the population as a whole varied from 0.089 to 0.785 over the loci and the average was 0.500 . The high levels of allelic diversity are coupled with very high $\mathrm{F}_{\text {is }}$ indicating that the population is experiencing high levels
TABLE 2: Bottleneck detection in the Jamunapari goats.

\begin{tabular}{lccccc}
\hline Marker & Heq $^{*}$ & SD & $*($ He-Heq $) / S D$ & He excess & M ratio* \\
\hline BM4621 & 0.894 & .021 & -1.825 & + & 0.833 \\
NRAMP & 0.834 & .036 & -0.886 & + & 0.692 \\
OarAE101 & 0.787 & .050 & 0.337 & - & 0.889 \\
IDVGA7 & 0.894 & .021 & -0.436 & + & 0.938 \\
ILSTS005 & 0.441 & .151 & 0.364 & - & 0.364 \\
BM6526 & 0.808 & .048 & -0.244 & + & 0.692 \\
ETH225 & 0.714 & .074 & -0.159 & + & 0.857 \\
OarHH56 & 0.813 & .043 & -0.007 & + & 0.857 \\
INRABERN192 & 0.833 & .038 & -0.359 & + & 0.625 \\
OarFCB48 & 0.784 & .053 & 0.845 & - & 1.00 \\
OarHH62 & 0.653 & .093 & 0.672 & - & 0.833 \\
TGLA40 & 0.831 & .041 & -1.275 & + & 0.769 \\
BM143 & 0.786 & .053 & -0.934 & + & 0.667 \\
SRCRSP5 & 0.786 & .052 & 0.051 & - & 0.800 \\
SRCRSP6 & 0.830 & .037 & -4.182 & + & 0.909 \\
SRCRSP9 & 0.849 & .034 & 0.763 & - & 0.846 \\
SRCRSP10 & 0.813 & .041 & 0.393 & - & 1.000 \\
\hline
\end{tabular}

${ }^{*} \mathrm{Heq}$ is the heterozygosity expected at equilibrium obtained through coalescent simulation under the "two-phase mutation model". (He-Heq)/SD: the standardized difference for each locus, $\mathrm{M}$ ratio: the number of allele/(range in allele size +1$)$.

of nonrandom mating in the breeding tract but simultaneously maintaining allelic diversity over the entire range of the breed. Gour et al. [31] also observed high inbreeding in Jamunapari goats; further, high estimates of inbreeding have been reported for Asian goat populations by Barker et al. [8].

Genetic variation at CSN1S1 and $\beta$-LG was 0.395 and 0.107 . The effective number of alleles was 1.653 and 1.20 at CSN1S1 and $\beta$-LG loci, respectively. The $\beta$-LG locus showed significant departure from HW equilibrium. The gene diversity and effective number of alleles at milk protein loci were higher than for 10 other Indian goat breeds, supporting the fact that the breed maintains higher genetic variability [18, 19].

The population was examined for allele frequency distribution for gaps, and $\mathrm{M}$ ratios are presented in Table 2. The $\mathrm{M}$ ratios ranged from 0.364 to 1.00 with an average of 0.815 , which was significantly higher than the critical value. The M ratio was less than 0.71 , diagnostic value of genetic bottlenecks, in the case of ILSTS005, INRABERN192, and BM143. The observed $\mathrm{M}$ ratio for all other markers in the population was very high and close to one indicating that it is a very slowly changing population (at least not showing the sign of bottleneck).

Bottleneck detection in Jamunapari goat was presented in Table 2. The mode shift test did not detect any distortion of allele frequency and showed a normal "L" shaped distribution which is a typical property of a population in equilibrium (Figure 2). The heterozygosity excess method was carried out to analyse historical bottlenecks. Out of 17 loci, 7 loci showed heterozygosity excess (Wilcoxon signed rank test, $P=0.8487$, one tail for heterozygosity excess), and there 


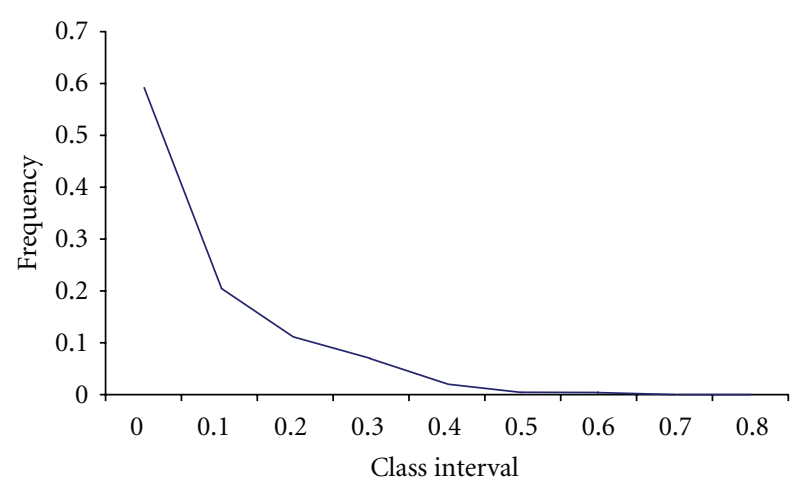

FIGURE 2: L-shaped mode shift graph showing the absence of bottleneck in Jamunapari goats.

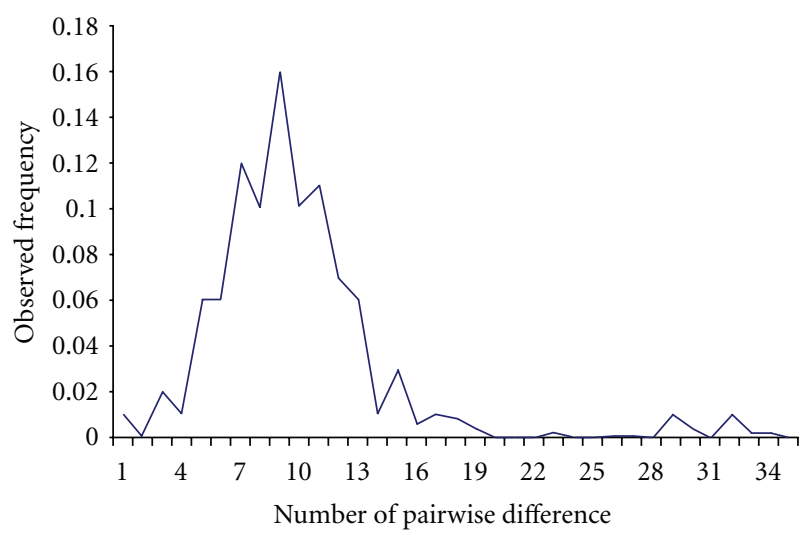

Figure 3: Mismatch distribution in Jamunapari goats analysed for mt-DNA control region.

was no significant departure from mutation-drift equilibrium detected in the population. Under the two-phase mutation model equilibrium, each individual has a roughly equal chance of having heterozygote deficiency or excess. The analysis indicated that the population has not suffered any bottleneck recently and was a constant size population. The present study agrees well with the observation of Gour et al. [31].

The sequence analysis of the $457 \mathrm{bp}$ mitochondrial HVRI region identified $34 \mathrm{mtDNA}$ haplotypes, and the overall haplotype diversity was 0.984 . The $\mathrm{mtDNA}$ variation detected relatively high number of haplotypes from a total 50 individuals, and haplotype diversity was quite high. Fu's Fs value is based on the probability of recovering a number of haplotypes greater than or equal to the observed number in a sample drawn from a stationery population with the same mean number of pairwise differences as the observed sample. Fu's Fs value was -15.53 . The significant negative Fs values indicated the large and sudden expansion in the population at geographical locations. The population showed a significant negative Fs value indicating excess of rare mutations, a pattern commonly attributed to a normal growing population.

Mismatch distribution analysis revealed the genetic signature of population expansion of Jamunapari goat. The tau value with $95 \%$ confidence interval was 8.20 (5.22-10.68), and the Sum of Square Difference (SSD) value was 0.0048 . The SSD value showed large population expansion in Jamunapari goats. Figure 3 depicts the mismatch distribution of Jamunapari goat. The shape of the distribution of number of observed differences between pairs of DNA sequences showed almost unimodal curve for the Jamunapari goat. The curve showed very small second mode towards the end indicating that some minor population expansion at some stage might have occurred in various geographical areas. Mismatch distribution has been extensively used to estimate the demographic parameters of past population expansion or contraction as it leaves a recognizable signature in the pattern of molecular diversity $[21,32,33]$. The unimodal distribution of pairwise differences in the breed (Figure 3 ) and Fu's Fs value indicated a sudden demographic population expansion and origin of the breed from a limited number of founder populations. Migration had an effect on the shape of curve, and intermediate migration rate would have led to a multimodal curve [34]. However, the factors like inbreeding and admixture with other population could affect the shape of mismatch distribution.

The amplified product for amelogenin gene (AMELY), SRY gene, and ZFY gene was $733 \mathrm{bp}, 632 \mathrm{bp}$, and $584 \mathrm{bp}$, respectively. No diversity was observed in SRY and AMELY genes. Nucleotide diversity and expected heterozygosity were $0.135 \pm 0.089$ and 0.1384 , respectively, for ZFY gene. Similarly Tajima's D value and Fu's Fs value were zero for SRY and AMELY gene. Tajima's D value and Fu's Fs value were 0.6797 and 2.539, respectively and non-significant for ZFY gene. Transition and transversion ratio was 47:90 for ZFY gene. ZFY gene showed low gene diversity and positive Tajima's D value and Fu's Fs value. Y-linked nucleotide diversity was found low in human, wolf, cattle, reindeer and Lynx indicating that reduced levels of Y-chromosome polymorphism may be a generalized feature of mammalian genome [35]. Y chromosome variability is expected to be lowest as compared to autosomes and $\mathrm{X}$-chromosome. The major factors explain that the low levels of Y chromosome variability are selection, mating system, or migration patterns, or other mechanisms lowering male effective population size. The low levels of $Y$ chromosome variability that we found in goat could be attributed to a strong sex bias in breeding.

The proportion of membership of individuals in the Jamunapari population placed $12.6 \%$ of population into one cluster group in both STRUCTURE analyses $(k=2$ or 3$)$. The cluster defined by STRUCTURE showed clear membership of individuals in two clusters indicating a genetic subdivision within the breed. There was a difference between expected and observed heterozygosity supporting the genetic subdivision within the breed. It has been also observed during survey that there are two strains of Jamunapari locally known as "Kathey" and "Kandhan". Kathey-type goats have long thick broad and less folded ears with thick and flat neck. Kandhan-type goats have long, soft, and folded ears, and the neck is thin and cylindrical. Kandhan is restricted to river banks of Chambal and Kathey is distributed throughout the breeding tract. (CIRG Report).

The average $\mathrm{M}$ ratio was large and above the critical significance value $(\mathrm{Mc})$ in the population suggesting that it has 
not suffered severe or long-lasting genetic bottlenecks [25, $36]$. Heterozygosity excess test and the mode shift indicators demonstrated that there was no sign of recent reductions in effective population size $(\mathrm{Ne})$ in the population. Moreover, the milk protein loci also exhibited higher gene diversity at both the loci as compared to other Indian goat breeds [18, 19]. Moreover, the mtDNA analysis supported population expansion model as evidenced from Fu's Fs value, unimodal distribution of pairwise differences (mismatch curve). Genetic variation is often reduced due to demographic reduction in population and can also show other signs of population bottlenecks. However, this breed showed retention of diversity in the face of population reduction. A similar type of trend had been observed in salmon population [37] and turtle [38]. Genetic responses to bottlenecks depend on life history and the life-span of the species, the severity of the demographic decline, level of present gene flow, and nature of demographic rebound $[25,39]$. Several factors might be affecting the population leading to maintenance of dramatic genetic variation despite severe demographic declines. The variation may be due to exchange of animals between different areas and selection criteria of farmers to use the individuals as parents for the next generation. The dispersal of local populations between farmers and selling to outside agencies may have served to maintain genetic variability in the population. The breeding buck is available with one or two farmers in every village and all the farmers mate their goats with the available bucks showing the nonrandom mating in the breeding tract but maintaining allelic diversity over the entire range of the breed. Again the existence of genetic subdivision supports the existing gene diversity in the population. Historically there was no evidence of a major earthquake or climate change in the area. Theoretically, the impact of even severe bottlenecks can be small if the bottleneck is followed by the rapid flush of growth in which most genetic variability is maintained [39]. Again, the population growth is evident but also animals are supplied to outside the home tract as a medium of remunerative income to farmers. Additionally, the overlapping generations in domestic goats buffer them from long-term losses of genetic variability in comparison to species with discrete generations. More importantly, the high reproductive rate of this large goat breed slows down the losses due to genetic drift from the base population. Small populations are generally considered to be susceptible to a number of genetic problems like low level of variability, inbreeding depression, and the ability to overcome disease agents; however, the population did not exhibit any such effect over the years in the adopted villages [12, 13]. The population has not shown any noticeable physiological sign of inbreeding depression as there was no reduction in fecundity (kidding rate); and the mortality in the breeding tract over the year was also low $(<7.5 \%,[12])$. It was observed that the rate of decline in genetic diversity was relatively slow in the population in its natural breeding tract; however, the low genetic diversity in $\mathrm{Y}$ chromosomal genes, as well as the effects of genetic bottlenecks in some loci due to decreased heterozygosity and lower level of $\mathrm{M}$ ratio, supports population reduction in breeding tract. Conservation will be much more difficult when the population becomes genetically impoverished and is effective and easy to implement when the populations are genetically stable. Therefore, it is necessary to initiate necessary steps to conserve the breed for future use.

\section{Acknowledgment}

The authors are grateful to the Director of Central Institute for Research on Goats for providing facilities to carry out the work.

\section{References}

[1] J. C. Avise, "Mitochondrial DNA polymorphism and a connection between genetics and demography of relevance to conservation," Conservation Biology, vol. 9, no. 3, pp. 686-690, 1995.

[2] D. W. Coltman, W. D. Bowen, and J. M. Wright, "Birth weight and neonatal survival of harbour seal pups are positively correlated with genetic variation measured by microsatellites," Proceedings of the Royal Society B, vol. 265, no. 1398, pp. 803809, 1998.

[3] D. H. Reed and R. Frankham, "Correlation between fitness and genetic diversity," Conservation Biology, vol. 17, no. 1, pp. 230237, 2003.

[4] E. H. Harley, I. Baumgarten, J. Cunningham, and C. O'Ryan, "Genetic variation and population structure in remnant populations of black rhinoceros, Diceros bicornis, in Africa," Molecular Ecology, vol. 14, no. 10, pp. 2981-2990, 2005.

[5] I. Tapio, S. Värv, J. Bennewitz et al., "Prioritization for conservation of northern European cattle breeds based on analysis of microsatellite data," Conservation Biology, vol. 20, no. 6, pp. 1768-1779, 2006.

[6] J. Kantanen, I. Olsaker, L.-E. Holm et al., "Genetic diversity and population structure of 20 North European cattle breeds," Journal of Heredity, vol. 91, no. 6, pp. 446-457, 2000.

[7] T. Pastor, J. C. Garza, P. Allen, W. Amos, and A. Aguilar, "Low genetic variability in the highly endangered mediterranean monk seal," Journal of Heredity, vol. 95, no. 4, pp. 291-300, 2004.

[8] J. S. F. Barker, S. G. Tan, S. S. Moore, T. K. Mukherjee, J. L. Matheson, and O. S. Selvaraj, "Genetic variation within and relationships among populations of Asian goats (Capra hircus)," Journal of Animal Breeding and Genetics, vol. 118, no. 4, pp. 213-233, 2001.

[9] M. H. Li, S. H. Zhao, C. Bian et al., "Genetic relationships among twelve Chinese indigenous goat populations based on microsatellite analysis," Genetics Selection Evolution, vol. 34, no. 6, pp. 729-744, 2002.

[10] M. B. Joshi, P. K. Rout, A. Mandal, K. Thangaraj, C. TylerSmith, and L. Singh, "Phylogeography and origin of Indian domestic goats," Molecular Biology and Evolution, vol. 21, no. 3, pp. 454-462, 2004.

[11] P. K. Rout, M. B. Joshi, A. Mandal, D. Laloe, L. Singh, and K. Thangaraj, "Microsatellite-based phylogeny of Indian domestic goats," BMC Genetics, vol. 9, article 11, 2008.

[12] P. K. Rout, V. K. Saxena, B. U. Khan et al., "Characterization of Jamunapari goats in their hometract," Animal Genetic Resource Information, vol. 27, pp. 43-53, 2000. 
[13] P. K. Rout, M. K. Singh, R. Roy, N. Sharma, and G. F. W. Haenlein, "Jamunapari-a diary goat breed in India," Dairy Goat Journal, vol. 82, no. 3, pp. 37-39, 2004.

[14] "World watch list for domestic animal diversity," in World Watch List, B. D. Scherf, Ed., FAO, Rome, Italy, 2000.

[15] M. Raymond and F. Rousset, "GENEPOP (version 1.2): population genetics software for exact tests and ecumenicism," Journal of Heredity, vol. 86, no. 3, pp. 248-249, 1995.

[16] J. Goudet, "FSTAT (version 1.2): a computer program to calculate F-statistics," Journal of Heredity, vol. 86, no. 6, pp. 485486, 1995.

[17] E. H. Harley, $A G A R_{S T}$, Version 2.8, A Program for Calculating Allele Frequencies, GST and $R_{S T}$ from Microsatellite Data. Wild Life Genetics Unit 2002, University of Care Town, Johannesburg, South Africa, 2002.

[18] A. Kumar, P. K. Rout, A. Mandal, and R. Roy, "Identification of the CSN1S1 allele in Indian goats by the PCR-RFLP method," Animal, vol. 1, no. 8, pp. 1099-1104, 2007.

[19] A. Kumar, P. K. Rout, and R. Roy, "Polymorphism of $\beta$-lacto globulin gene in Indian goats and its effect on milk yield," Journal of Applied Genetics, vol. 47, no. 1, pp. 49-53, 2006.

[20] F. Yeh, R.-C. Yang, and T. Boyle, POPGENE Version 1.31, University of Alberta, Edmonton, Canada, 1999.

[21] A. R. Rogers and H. Harpending, "Population growth makes waves in the distribution of pairwise genetic differences," Molecular Biology and Evolution, vol. 9, no. 3, pp. 552-569, 1992.

[22] L. Excoffier, P. Smouse, and J. Quattro, "Analysis of molecular variance inferred from metric distances among DNA haplotypes: application to human mitochondrial DNA restriction data," Genetics, vol. 131, no. 2, pp. 479-491, 1992.

[23] X.-Y. Fu, "Statistical tests of neutrality of mutations against population growth, hitchhiking and background selection," Genetics, vol. 147, no. 2, pp. 915-925, 1997.

[24] J. R. S. Meadows, R. J. Hawken, and J. W. Kijas, "Nucleotide diversity on the ovine Y chromosome," Animal Genetics, vol. 35, no. 5, pp. 379-385, 2004.

[25] J. C. Garza and E. Williamson, "Detection of reduction in population size using data from microsatellite loci," Molecular Ecology, vol. 10, no. 2, pp. 305-318, 2001.

[26] S. Piry, G. Luikart, and J. M. Cornuet, "BOTTLENECK: a computer program for detecting recent reductions in the effective population size using allele frequency data," Journal of Heredity, vol. 90, no. 4, pp. 502-503, 1999.

[27] J. M. Cornuet and G. Luikart, "Description and power analysis of two tests for detecting recent population bottlenecks from allele frequency data," Genetics, vol. 144, no. 4, pp. 2001-2014, 1996.

[28] G. L. Luikart, F. W. Allendorf, J. M. Cornuet, and W. B. Sherwin, "Distortion of allele frequency distributions provides a test for recent population bottlenecks," Journal of Heredity, vol. 89, no. 3, pp. 238-247, 1998.

[29] A. Di Rienzo, A. C. Peterson, J. C. Garza, A. M. Valdes, M. Slatkin, and N. B. Freimer, "Mutational processes of simplesequence repeat loci in human populations," Proceedings of the National Academy of Sciences of the United States of America, vol. 91, no. 8, pp. 3166-3170, 1994.

[30] J. K. Pritchard, M. Stephens, and P. Donnelly, "Inference of population structure using multilocus genotype data," Genetics, vol. 155, no. 2, pp. 945-959, 2000.

[31] D. S. Gour, G. Malik, S. P. S. Ahlawat et al., "Analysis of genetic structure of Jamunapari goats by microsatellite markers," Small Ruminant Research, vol. 66, no. 1-3, pp. 140-149, 2006.
[32] L. Excoffier and S. Schneider, "Why hunter-gatherer populations do not show signs of Pleistocene demographic expansions," Proceedings of the National Academy of Sciences of the United States of America, vol. 96, no. 19, pp. 10597-10602, 1999.

[33] L. M. Vigilant, M. Stoneking, H. Harpending, K. Hawkes, and A. C. Wilson, "African populations and the evolution of human mitochondrial DNA," Science, vol. 253, no. 5027, pp. 1503-1507, 1991.

[34] P. Marjoram and P. Donnelly, "Pairwise comparisons of mitochondrial DNA sequences in subdivided populations and implications for early human evolution," Genetics, vol. 136, no. 2, pp. 673-683, 1994.

[35] L. Hellborg and H. Ellegren, "Low levels of nucleotide diversity in mammalian Y chromosomes," Molecular Biology and Evolution, vol. 21, no. 1, pp. 158-163, 2004.

[36] K. C. Doerner, W. Braden, J. Cork et al., "Population genetics of resurgence: white-tailed deer in Kentucky," Journal of Wildlife Management, vol. 69, no. 1, pp. 345-355, 2005.

[37] H. Neville, D. Isaak, R. Thurow, J. Dunham, and B. Rieman, "Microsatellite variation reveals weak genetic structure and retention of genetic variability in threatened Chinook salmon (Oncorhynchus tshawytscha) within a Snake River watershed," Conservation Genetics, vol. 8, no. 1, pp. 133-147, 2007.

[38] C. H. Kuo and F. J. Janzen, "Genetic effects of a persistent bottleneck on a natural population of ornate box turtles (Terrapene ornata)," Conservation Genetics, vol. 5, no. 4, pp. 425437, 2004.

[39] M. Nei, T. Maruyama, and R. Chakraborty, "The bottleneck effect and genetic variability in populations," Evolution, vol. 29, no. 1, pp. 1-10, 1975. 

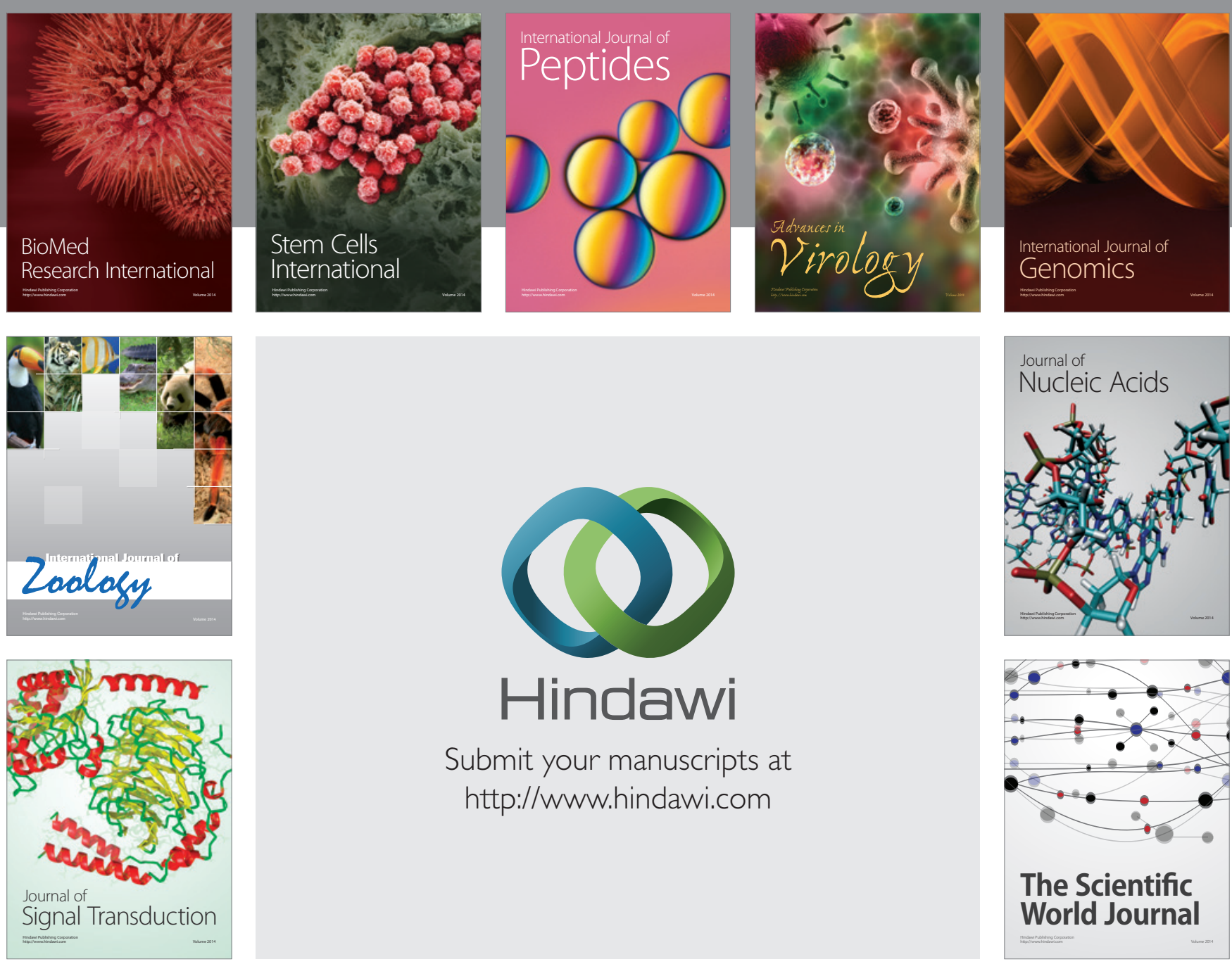

Submit your manuscripts at

http://www.hindawi.com
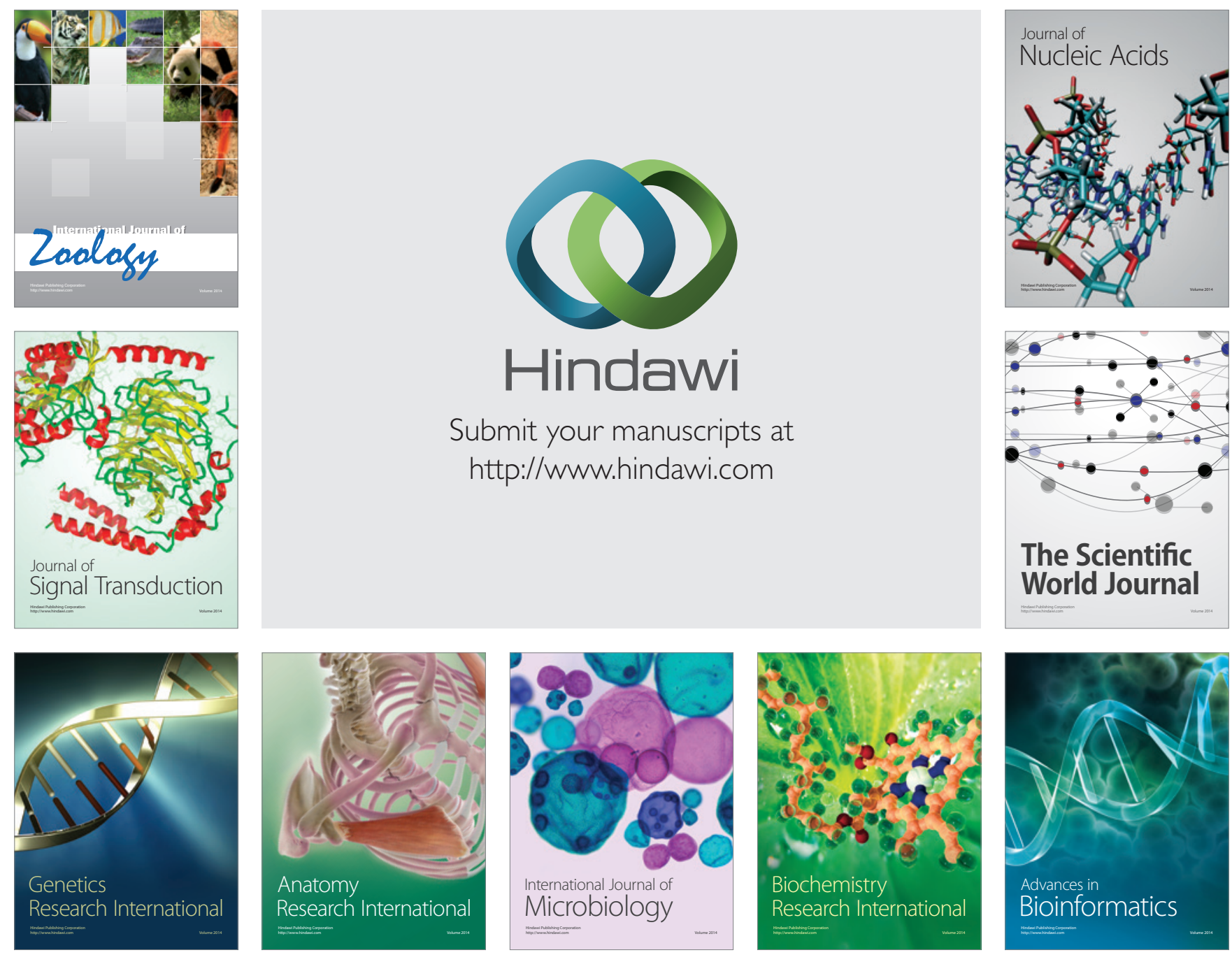

The Scientific World Journal
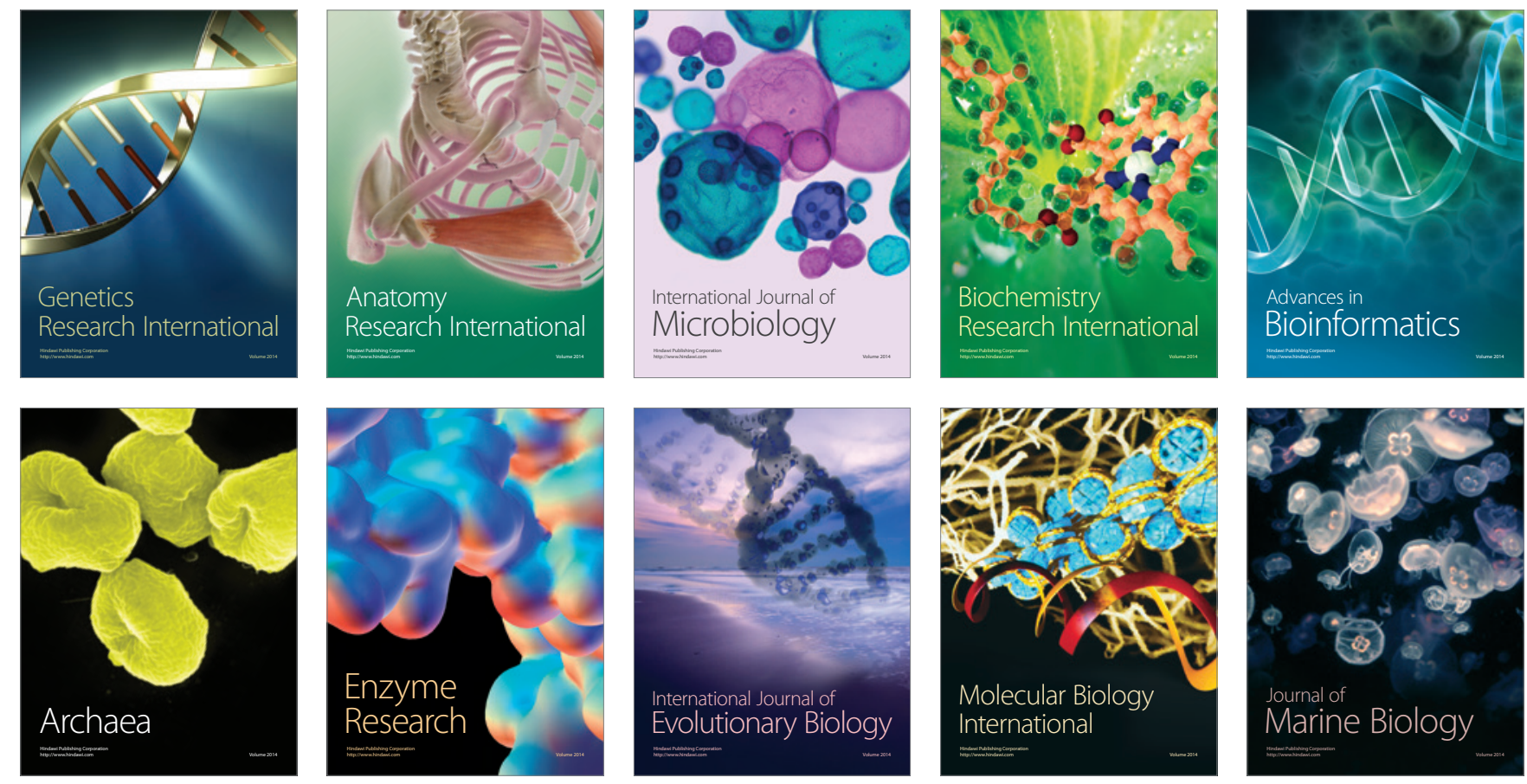